\title{
PORCIONAMENTO, CONSUMO E DESPERDÍCIO EM UM RESTAURANTE ESCOLAR
}

\section{PORTIONING, CONSUMPTION, AND WASTE IN A SCHOOL CAFETERIA}

\section{Nathália Bernardo Faquim ${ }^{1}$ Telma de Oliveira ${ }^{2}$ Mônica Glória Neumann Spinelli ${ }^{3}$}

RESUMO: A qualidade e quantidade dos alimentos ingeridos pela criança são essenciais, nos primeiros anos de vida, e acarretam repercussões nas práticas alimentares, ao longo desta. O objetivo deste estudo foi avaliar, durante cinco dias, o porcionamento de alimentos, o consumo médio e o desperdício, em um restaurante de unidade escolar particular do município de São Paulo, com crianças de 5 a 6 anos, em 2011. O consumo alimentar variou entre 120,8 e 243,7g, a porcentagem de sobras de saladas entre 35,9 e 97,5\%, e os restos entre 22,6 e 49,1\%. Concluiu-se que o porcionamento variou de acordo com o funcionário que prepara o prato e não de acordo com o apetite ou vontade da criança. É importante que, além do conhecimento do per capita próprio, para essa faixa etária, estabeleça-se um sistema de self service para que a criança crie o seu próprio padrão de consumo.

Palavras-chave: criança; consumo; alimentação escolar.

ABSTRACT: The quality and quantity of food consumed by child are essential in the first years of life and have long-term repercussions in eating habits. The aim of this study was to evaluate the portioning of food as well as the average consumption and waste in a restaurant of a private school in São Paulo of children 5-6 years old, during five days in 2011. Food consumption varied between 120.8 and $243.7 \mathrm{~g}$. The percentage of salad leftovers was between 35.9 and $97.5 \%$ and other foods between 22.6 and $49.1 \%$. It was concluded that the portioning varied according to the person who prepared the dish and not according to the appetite or desire of the child. In addition to information about the per capita suitable for this age group, it is important to establish a self-service system for the children to create their own consumption pattern.

Keywords: children; food consumption; school cafeteria.

${ }^{1}$ Graduada em Nutrição - Universidade Paulista - UNIP. E-mail: nathfaquim@gmail.com.

${ }_{2}$ Graduada em Nutrição - UNIP e Nutricionista da Associação Brasileira de Nutrição - ASBRAN. E-mail: telmaoliveira87@gmail.com.

${ }^{3}$ Doutora em Saúde Pública - Universidade de São Paulo - USP e professora titular da UNIP. E-mail: spinelli@usp.br. 


\section{INTRODUÇÃO}

A nutrição humana é de suma importância do ponto de vista da saúde e do bem-estar das populações, (FARIAS JUNIOR; OSÓRIO, 2005), e os primeiros anos de vida da criança são fundamentais ao seu bom desenvolvimento, caracterizando-se por uma alta velocidade de crescimento. Nessa fase, a qualidade e quantidade dos alimentos ingeridos têm papel fundamental, nesses dois aspectos, e acarreta repercussões nas práticas alimentares ao longo da vida (CONCELIER et al., 2009).

Apesar da alimentação ser essencial, em qualquer fase da vida do ser humano, é nos primeiros anos de vida que desempenha papel decisivo (GOULART et al., 2003). A formação do hábito alimentar acontece na infância; por isso, é necessário o entendimento dos seus fatores determinantes, para que seja possível sugerir processos educativos efetivos para obter mudanças no padrão alimentar da criança (RAMOS; STEIN, 2000).

Segundo Vitolo et al. (2005), as possibilidades de mudanças das práticas alimentares de crianças menores podem ser limitadas por várias condições, encontrandose vinculadas tanto à disponibilidade de alimentos quanto à sua escolha, dependendo dos valores culturais e das bases educacionais.

Os guias dietéticos para crianças têm se difundido, tendo como foco tanto a desnutrição e deficiências nutricionais, como o sobrepeso em crianças. Esses guias têm sido utilizados para descrever as recomendações quantitativas e qualitativas dos padrões dietéticos, padrões estes baseados em evidências científicas. $O$ Departamento de Agricultura dos Estados
Unidos (United States Department of Agriculture (USDA) desenvolveu a Pirâmide Alimentar para crianças. Essa pirâmide é conhecida como uma ferramenta de educação nutricional e recomenda que a dieta contenha o número de porções adequadas dos cinco grupos de alimentos (cereais, vegetais, fruta, carnes, leite) (BARBOSA et al., 2006).

Atualmente, tem aumentado o número de escolas que oferecem almoço para as crianças que cumprem período integral, e, embora as deficiências alimentares em crianças levem ao crescimento deficiente, aumentem a vulnerabilidade às infecções e promovam deficiências no processo de maturação do sistema nervoso e no desenvolvimento mental e intelectual (LODI; GATTI, 2009), a literatura que trata das questões de porcionamento e de consumo para as faixas etárias menores ainda é escassa. A identificação e a padronização do porcionamento, bem como a avaliação do desperdício são de fundamental importância para oferecer melhor qualidade de serviço e maior atenção nutricional (ABREU; SPINELLI; SOUZA PINTO, 2011; NONINOBORGES et al., 2006).

Dessa forma, o objetivo deste estudo foi identificar o porcionamento de alimentos, o consumo médio e o desperdício em um restaurante de unidade escolar particular.

\section{MÉTODOS}

A população de estudo foram crianças de 4 a 5 anos que estudam em um colégio privado do município de São Paulo.

Durante cinco dias, em outubro de 2011, foram pesadas as porções de arroz, feijão, prato protéico e guarnição oferecidos às crianças. Os pratos são porcionados 
pelas funcionárias que trabalham no refeitório. As saladas são servidas, no prato, conforme a vontade da criança; por isso, foram pesadas antes e depois da distribuição (total preparado - sobras) e o total oferecido foi dividido pelo número de crianças, obtendo-se, dessa forma, o per capita médio, segundo descrito em Abreu, Spinelli e Souza Pinto (2011). Após o almoço das crianças, todo o resto foi pesado e anotado, assim como a quantidade de crianças que consumiram a refeição.

O consumo foi considerado o peso da porção menos a porcentagem de resto. $O$ resto foi calculado dividindo-se o peso total do descarte dos pratos pelo número de crianças. $\mathrm{Na}$ impossibilidade de separar $\mathrm{o}$ resto por tipo de alimento, foi estabelecido que as quantidades de cada componente estariam presentes no lixo nas mesmas proporções em que se encontravam no momento da distribuição. Avaliadas as proporções de cada alimento, nos restos, estimaram-se por regra de três, as quantidades consumidas.

Para a realização da pesagem, utilizou-se uma balança da marca Filizola, tomando-se os cuidados higiênico-sanitários adequados. Após a montagem dos pratos, a porção de cada alimento foi pesada, separadamente, anotando-se cada resultado obtido.
Foi solicitada a autorização e 0 preenchimento do Termo de Conhecimento Consentido para a realização da pesquisa.

\section{RESULTADOS E DISCUSSÃO}

Pode-se observar, no Quadro 1, a variabilidade do tamanho das porções, que dependem da pessoa que está servindo o alimento, e do utensílio utilizado.

As funcionárias do restaurante porcionam os alimentos, nos pratos, com exceção da salada - que é oferecida, individualmente, a cada criança - podendo se observar que cada dia as quantidades variam, com exceção do feijão, que tem o peso, padronizado, de 80 gramas, por ser servido com concha cheia.

O cardápio apresentado no Quadro 1 mostra uma adequação em relação à qualidade nutricional das guarnições, pois, na maior parte dos dias, foram servidos vegetais preparados sem a utilização de frituras. Segundo Fox et al. (2009), entre escolares do ensino fundamental, a oferta, por mais de uma vez, por semana, de batatas fritas e produtos similares, na alimentação escolar, está associada, significativamente, ao aumento de obesidade. 


\section{Quadro 1 - Peso da porção servida, quantidade de consumidores e consumo per capita. São Paulo, 2011}

\begin{tabular}{|c|c|c|c|}
\hline Dia & $\begin{array}{c}\text { Peso da Porção Servida } \\
\text { (g) }\end{array}$ & $\begin{array}{l}\text { Total de } \\
\text { crianças }\end{array}$ & $\begin{array}{c}\text { Consumo } \\
\text { (g) }\end{array}$ \\
\hline \multirow{7}{*}{1} & Arroz $=55$ & \multirow{7}{*}{65} & 42,6 \\
\hline & Feijão $=80$ & & 62,0 \\
\hline & Filé de Alcatra $=30$ & & 23,2 \\
\hline & Filé de Chester $=30$ & & 23,2 \\
\hline & Abobrinha $=45$ & & 34,8 \\
\hline & Acelga $=20$ & & 15,5 \\
\hline & Saladas $=54,7$ & & 42,4 \\
\hline \multirow{7}{*}{2} & Arroz $=55$ & \multirow{7}{*}{71} & 41,0 \\
\hline & Feijão $=80$ & & 63,6 \\
\hline & Maminha $=25$ & & 18,6 \\
\hline & Frango $=25$ & & 18,6 \\
\hline & Rondele $=50$ & & 37,3 \\
\hline & Espinafre $=15$ & & 11,2 \\
\hline & Saladas $=34,7$ & & 25,9 \\
\hline \multirow{7}{*}{3} & Arroz $=35$ & \multirow{7}{*}{65} & 24,8 \\
\hline & Feijão $=80$ & & 56,8 \\
\hline & Kibe de forno $=25$ & & 17,7 \\
\hline & Coxa chapeada $=20$ & & 14,2 \\
\hline & Seleta de legumes $=20$ & & 14,2 \\
\hline & Couve $=10$ & & 7,1 \\
\hline & Saladas $=52,4$ & & 37,2 \\
\hline \multirow{7}{*}{4} & Arroz $=50$ & \multirow{7}{*}{67} & 36,4 \\
\hline & Feijão $=80$ & & 58,2 \\
\hline & Filé Mignon $=15$ & & 10,9 \\
\hline & Filé de frango $=20$ & & 14,5 \\
\hline & Risoto de brócolis $=25$ & & 18,2 \\
\hline & Escarola $=10$ & & 7,3 \\
\hline & Saladas $=28,7$ & & 20,9 \\
\hline \multirow{7}{*}{5} & Arroz $=35$ & \multirow{7}{*}{67} & 18,5 \\
\hline & Feijão $=80$ & & 42,3 \\
\hline & Peixe $=20$ & & 10,6 \\
\hline & Filé de Alcatra $=20$ & & 10,6 \\
\hline & Cuscuz a paulista $=30$ & & 15,9 \\
\hline & Espinafre $=15$ & & 7,9 \\
\hline & Saladas $=28,4$ & & 15,0 \\
\hline
\end{tabular}

Observa-se, no Quadro 2, que as porcentagens de restos foram muito altas, variando entre 22,6 e $47,1 \%$. Para Campos, Viana e Rocha (2011), que avaliaram desperdícios alimentares em meio escolar, encontrando $31 \%$ de restos, estes refletem a falta de integração com o cliente. No dia 5, a porcentagem de resto foi bem maior, comparada as demais, e o consumo foi, praticamente, $50 \%$ inferior ao dia de maior consumo. O resto é um importante avaliador da qualidade da comida ou da adequação do tamanho da porção servida, em caso de restaurantes com serviço self-service
(ABREU; SPINELLI; SOUZA PINTO, 2011; $V A Z, 2006)$. Nesse caso, em que a criança é servida, é preciso estar muito atento ao tipo de alimento deixado no prato e nos comentários das crianças para saber o real motivo do baixo consumo - alimento não habitual da criança, modo de preparo, tipo de corte, tempero etc. Segundo Abreu, Spinelli e Souza Pinto (2011), o resto deve ser algo muito próximo do zero. Os autores referem que os restos podem ocorrer por diversos motivos, como alimentos que não atenderam às características sensoriais esperadas, 0 porcionamento excessivo, por parte de 
colaborador ou cliente, dentre outros.

De acordo com Teixeira et al. (1990), não existem valores para Indicador de Restos (IR), quando a população-alvo são crianças e/ ou jovens, o valor utilizado será o estipulado para a população adulta saudável (10\%). No geral, os resultados apresentados, em relação à porcentagem de resto, estão altos.

\section{Quadro 2 - Peso da porção servida, porcentagem de resto e porção final. São Paulo, 2011}

\begin{tabular}{|c|c|c|c|}
\hline Dia & $\begin{array}{c}\text { Peso da Porção Servida } \\
\text { (g) }\end{array}$ & $\begin{array}{c}\text { Resto } \\
(\%)\end{array}$ & $\begin{array}{c}\text { Porção Final } \\
\text { (g) }\end{array}$ \\
\hline 1 & $\begin{array}{l}\text { Arroz }=55 \\
\text { Feijão }=80 \\
\text { Filé de Alcatra }=30 \\
\text { Filé de Chester }=30 \\
\text { Abobrinha }=45 \\
\text { Acelga }=20 \\
\text { Saladas }=54,7\end{array}$ & 22,6 & 243,7 \\
\hline 2 & $\begin{array}{l}\text { Arroz }=55 \\
\text { Feijão }=80 \\
\text { Maminha }=25 \\
\text { Frango }=25 \\
\text { Rondele }=50 \\
\text { Espinafre }=15 \\
\text { Saladas }=34,7 \\
\end{array}$ & 25,5 & 212,1 \\
\hline 3 & $\begin{array}{l}\text { Arroz }=35 \\
\text { Feijão }=80 \\
\text { Kibe de forno }=25 \\
\text { Coxa chapeada }=20 \\
\text { Seleta de legumes }=20 \\
\text { Couve }=10 \\
\text { Saladas }=52,5 \\
\end{array}$ & 29,0 & 172,1 \\
\hline 4 & $\begin{array}{l}\text { Arroz }=50 \\
\text { Feijão }=80 \\
\text { Filé Mignon }=15 \\
\text { Filé de frango }=20 \\
\text { Risoto de brócolis }=25 \\
\text { Escarola }=10 \\
\text { Saladas }=28,7 \\
\end{array}$ & 27,3 & 166,3 \\
\hline 5 & $\begin{array}{l}\text { Arroz }=35 \\
\text { Feijão }=80 \\
\text { Peixe }=20 \\
\text { Filé de Alcatra }=20 \\
\text { Cuscuz a paulista }=30 \\
\text { Espinafre }=15 \\
\text { Saladas }=28,4\end{array}$ & 47,1 & 120,8 \\
\hline
\end{tabular}

Pode ser observado, no Quadro 3, que a aceitação de saladas, em dois dos dias (4 e 5), foi muito baixa, comparada aos demais, possivelmente devido ao tipo de prepara-ções ofertados. É importante observar que, mesmo nos dias com maior aceitação, os valores ainda são baixos, evidenciados pelas altas quantidades de sobras durante todos os dias. As sobras identificam erros de planejamento da determinação do número de refeições, dos per capitas e de prepa-rações incompatíveis com o tipo de cliente (ABREU; SPINELLI; SOUZA PINTO, 2011). Como nesse caso, há pouca variação no número de crianças que fazem as refeições, ficam evidenciadas as questões referentes às quantidades preparadas e à adequação ao hábito alimentar, que pode ser observadas, por exemplo, no baixo consumo de preparações, como picles e nabo ralado com azeitonas 
pretas, que, além da inadequação sensorial, contêm grandes quantidades de sódio.

O consumo de hortaliças foi baixo, na maior parte dos dias. É possível observar, no Quadro 3, que, provavelmente, o tipo de corte tenha interferência na quantidade consumida, uma vez que o tomate cortado em cubos apresentou os maiores per capitas e, considerando que, nessa unidade, a quantidade de salada é determinada pela criança.

Robinson-O'Brien et al. (2010) concluíram, em seu estudo, que o consumo de FLV, por crianças escolares, encontravase em níveis abaixo do recomendado, principalmente entre as de nível socioeconômico mais baixo, e que a escola pode representar uma importante contribuição para o aumento desse consumo. Programas, no ambiente escolar, que promovam o aumento do consumo de FLV podem representar uma oportunidade de encorajamento para tal.

Segundo Proença (1997), um dos maiores desafios para o nutricionista é conseguir conciliar a alimentação saudável, os hábitos alimentares, características sensoriais, e modo de apresentação, todos ao mesmo tempo, na elaboração da ementa e na distribuição das refeições.

De acordo com Lytle et al. (1996), a intervenção alimentar, na escola, pode influenciar, positivamente, as crianças, principalmente no consumo de gordura total e saturada, sugerindo que uma abordagem dirigida a essa população pode ser efetiva para diminuir os fatores de risco, para doenças cardiovasculares, nessa faixa etária.

\section{Quadro 3 - Peso do total preparado de saladas, sobras, oferta e consumo per capita.} São Paulo, 2011

\begin{tabular}{|c|c|c|c|c|c|c|c|}
\hline Dia & Saladas & $\begin{array}{c}\text { Total } \\
\text { preparado }\end{array}$ & $\begin{array}{c}\text { Sobras } \\
\text { (g) }\end{array}$ & $\begin{array}{c}\text { Sobras } \\
(\%)\end{array}$ & $\begin{array}{l}\text { Oferta } \\
\text { (g) }\end{array}$ & $\begin{array}{l}\text { Per capita } \\
\text { (g) }\end{array}$ & $\begin{array}{c}\text { Per capita } \\
\text { total }\end{array}$ \\
\hline 1 & Buquê de folhas & 892 & 320 & 35,9 & 572 & 8,8 & \multirow{4}{*}{54,7} \\
\hline & Tomate em cubos & 2600 & 1045 & 40,2 & 1555 & 23,9 & \\
\hline & Milho Temperado & 2320 & 989 & 42,6 & 1331 & 20,5 & \\
\hline & Picles & 1650 & 1550 & 93,9 & 100 & 1,5 & \\
\hline \multirow[t]{4}{*}{2} & Alface Crespa & 900 & 340 & 37,8 & 560 & 7,9 & \multirow{4}{*}{34,7} \\
\hline & Tomate em rodelas & 2570 & 1695 & 65,9 & 875 & 12,3 & \\
\hline & Chuchu Mimoso & 1400 & 1100 & 78,6 & 300 & 4,2 & \\
\hline & Beterraba Cozida & 1820 & 1090 & 59,9 & 730 & 10,3 & \\
\hline \multirow[t]{4}{*}{3} & Alface Lisa & 896 & 245 & 27,3 & 651 & 10,0 & \multirow{4}{*}{52,4} \\
\hline & Tomate em cubos & 2540 & 1010 & 39,8 & 1530 & 23,5 & \\
\hline & Pepino em rodelas & 2230 & 1500 & 67,3 & 730 & 11,2 & \\
\hline & Mandioquinha em cubos & 1900 & 1400 & 73,7 & 500 & 7,7 & \\
\hline \multirow[t]{4}{*}{4} & Rúcula com tomate seco & 805 & 510 & 63,4 & 295 & 4,4 & \multirow{4}{*}{28,7} \\
\hline & Tomate em lâminas & 2565 & 1325 & 51,7 & 1240 & 18,5 & \\
\hline & Berinjela grelhada com mostarda & 1395 & 1285 & 92,1 & 110 & 1,6 & \\
\hline & Salada Veggie & 1830 & 1550 & 84,7 & 280 & 4,2 & \\
\hline \multirow[t]{4}{*}{5} & Salada Ceasar & 2400 & 1550 & 64,6 & 850 & 12,7 & \multirow{4}{*}{28,4} \\
\hline & Tomate em meia lua & 2460 & 2075 & 84,3 & 385 & 5,7 & \\
\hline & Nabo ralado com azeitonas pretas & 1405 & 1370 & 97,5 & 35 & 0,5 & \\
\hline & Salada Tropical & 2760 & 2125 & 77,0 & 635 & 9,5 & \\
\hline
\end{tabular}

\section{CONCLUSÃO}

Concluiu-se que o porcionamento varia de acordo com o funcionário que prepara 0 prato e não de acordo com o apetite ou vontade da criança. Para tal, é importante que, além do conhecimento do per capita próprio para o consumo, nessa faixa etária, estabeleça-se um sistema de self-service monitorado para que a própria criança crie o 
seu padrão de consumo, diminuindo, assim, a alta porcentagem de restos.

Há necessidade de se promover projetos de promoção de educação alimentar de modo a aumentar o consumo.

\section{REFERÊNCIAS}

ABREU, E. S.; SPINELLI, M. G. N.; SOUZA PINTO, A. M. Gestão de Unidades de Alimentação e Nutrição: um modo de fazer. 4. ed. São Paulo: Metha, 2011.

BARBOSA, S. M. R. et al. E. Avaliação do consumo alimentar de crianças pertencentes a uma creche filantrópica na llha de Paquetá, Rio de Janeiro, Brasil. Rev. bras. saúde matern. infant., v. 6, n. 1, p. 127-134. 2006.

CAMPOS, V.; VIANA, I.; ROCHA, A. Estudo de desperdícios alimentares em meio escolar. Nutr. pauta, v. 19, n. 109, p. 60-64. 2011.

CONCELIER, L. C. A. et al. Situação alimentar de crianças entre zero e dois anos atendidas em Programa de Saúde da Família no sul do estado de Santa Catarina. ACM arq. catarin. med., v. 38, n. 1, p. 20-25, 2009.

FARIAS JUNIOR, G.; OSÓRIO, M. M. Padrão alimentar de crianças menores de cinco anos. Rev. nutr., v. 18, n. 6, p. 793-802, 2005.

FOX, M. K. et al. Association between School Food Environment and Practices and Body Mass Index of US Public School Children. J. Am. Diet. Assoc., v. 109, n. 2, p. 108-117, 2009.

GOULART, M. M. R. et al. Avaliação do consumo alimentar de pré-escolares. Rev. bras. ciênc. saúde, v. 1, n. 2, p. 7-13, 2003.

LODI, A. L.; GATTI, R. R. Análise da ingestão diária e avaliação da qualidade nutricional das refeições consumidas pelas crianças, matriculadas em uma creche de cidade do interior do Paraná. In: III SEMANA ACADÊMICA DO CURSO DE NUTRIÇÃO DA UNICENTRO, 3., 2009, Guarapuava. Anais... Guarapuava: UNICENTRO, 2009. v. $1 . \quad$ Disponível em: $<$ http://anais.unicentro.br/senutri2009/pdf/res umo_33.pdf >. Acesso em: 22 maio 2012.

LYTLE, L. A. et al. Changes in nutrient intakes of elementary school children following a school based intervention: results from the CATCH study. Prev. med., v. 25, n. 4, p. 465-477, 1996.

NONINO-BORGES, C. B. et al. Desperdício de alimentos intra-hospitalar. Rev. Nutri., v. 19, n. 3, p. 349-356, 2006.

PROENÇA, R. P. C. Inovação Tecnológica na Produção de Alimentação Coletiva. Florianópolis: Insular, 1997.

RAMOS, M.; STEIN, M. L. Desenvolvimento do comportamento alimentar infantil. $J$. pediatr. (Rio J.)., v. 76, n. 3, p. 229-238, 2000.

ROBINSON-O'BRIEN, R. et al. Associations Between School Meals Offered Through the National School Lunch Program and the School Breakfast Program and Fruit and Vegetable Intake Among Ethnically Diverse, Low-Income Children. J. sch. health., v. 80, n. 10, p. 487- 492, 2010.

TEIXEIRA, S. M. F. G. et al. Administração Aplicada às Unidades de Alimentação e Nutrição. São Paulo: Atheneu, 1990.

VAZ, C. S. Restaurantes: controlando custos e aumentando lucros. São Paulo: Metha, 2006.

VITOLO, R. M. et al. Impactos da implementação dos dez passos da alimentação saudável para crianças: ensaio de campo randomizado. Cad. saúde pública, v. 21 , n. 5 , p. 1448-1457, 2005. 